\title{
Multi-area control of overlapping areas in power systems for FACTS control
}

\author{
Gabriela Hug-Glanzmann*, Rudy Negenborn ${ }^{+}$, Göran Andersson*, Bart De Schutter ${ }^{+, o}$, Hans Hellendoorn ${ }^{+}$ \\ * Power Systems Laboratory \\ + Delft Center for Systems and Control / \\ ETH Zürich \\ Zurich, Switzerland \\ $\{$ hug,andersson\}@eeh.ee.ethz.ch \\ ${ }^{o}$ Department of Marine and Transport Technology \\ Delft University of Technology \\ Delft, The Netherlands \\ r.r.negenborn@tudelft.nl
}

\begin{abstract}
As power systems generally are large interconnected systems controlled by several parties, centralized optimal power flow (OPF) control taking the entire grid into account is often not feasible. To use optimal control in power systems nevertheless, the overall system is decomposed into areas with associated subproblems, which are solved in an iterative way. Currently available decomposition techniques assume that the models and control objectives of areas are formulated to be non-overlapping, i.e., the border of one area is at the same time also the border of a neighboring area. However, when the areas are determined independently from each other, e.g., by sensitivity analysis, the areas can be overlapping, making currently existing techniques not directly applicable. In this paper, we extend one of these techniques, viz. a modified Lagrange decomposition method, to the case of overlapping areas. Simulations are carried out on an adjusted IEEE 57-bus system in which the controlled entities are FACTS devices and the objective is to improve system security.
\end{abstract}

\section{INTRODUCTION}

Optimal power flow (OPF) is a well known-method to control and optimize the operation of a power system [1]. Typically, a model of the considered power system is used to formulate an optimization problem to find the optimal settings of the controllable devices with respect to a given objective function and subject to given constraints. Such an approach assumes that a model of the power system is available and that settings can be determined from a central point in the system. However, for larger power systems, e.g., the Europe-covering UCTE grid, obtaining an accurate overall model of the system is cumbersome due to the system's size, and even if a model would be available, the computations to solve the optimization problem would become intractable. Moreover, large power systems typically span several countries or regions, each of them having control of only their own part of the power system, making implementation of central control unfeasible.

To facilitate the application of OPF to large-scale problems, the overall system can be decomposed into smaller areas, each with an associated control subproblem, which is solved iteratively in a coordinated way. This coordination is necessary, since a setting chosen in one area will influence the situation and thus the choice of settings in the other areas of the system.

Traditional approaches for multi-area OPF assume that a decomposition of the overall system model and control objectives is possible into models of areas that cover strictly

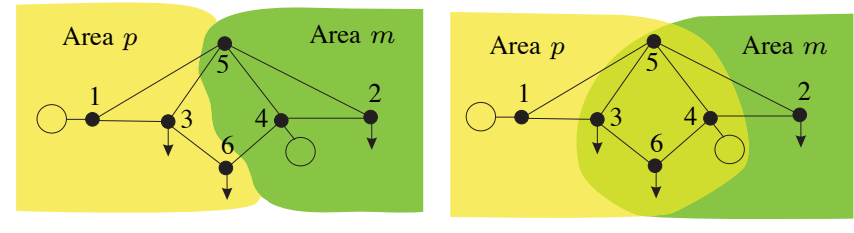

Fig. 1. Non-overlapping (left, no buses are shared) versus overlapping areas (right, some buses are shared).

separate regions [2], [3], i.e., the areas are assumed to be nonoverlapping, see Fig. 1. When OPF is used for power flow control of multiple areas, and each bus can be assigned only to one individual area, then this is an appropriate assumption. However, when sensitivity analysis is used to determined the minimal area that individual FACTS devices have to consider [4], then for FACTS devices that are close to each other, the corresponding areas will be overlapping, thus not satisfying the underlying assumption of current decomposition techniques. In the following, a particular approach for multi-area control, first proposed in [3] for non-overlapping areas, is extended and applied to the case of overlapping areas. The method is used to coordinate FACTS devices for steady-state system security by improving the voltage profile, preventing lines from overloading, and minimizing active power losses.

This paper is organized as follows. In Sect. II the models of the network and FACTS devices used for OPF are described. In Sect. III the overall OPF problem is defined. In Sect. IV current decomposition techniques which assume non-overlapping areas are discussed. In Sect. V an extension to overlapping areas for one of these methods is proposed, and in Sect. VI simulation results are presented for FACTS control of overlapping areas in an adjusted IEEE 57-bus system.

\section{MODELS FOR POWER SYSTEMS WITH FACTS DEVICES}

As the focus lies on improving the steady-state system security, the power system is modeled using static equations describing the steady-state characteristics of the power system. For the transmission lines the well known $\pi$-model is used [1] Generators are modeled with constant active power injection and constant voltage magnitude, while loads are modeled 


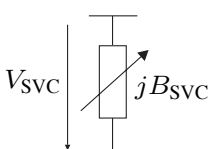

(a)

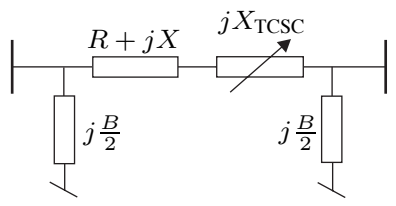

(b)
Fig. 2. (a) Model of an SVC and (b) of a TCSC.

with constant active and constant reactive power injections. A single generator is used as slack generator with fixed voltage magnitude and angle [1]. The FACTS devices that we consider are Static Var Compensators (SVCs) and Thyristor Controlled Series Compensators (TCSCs), since these FACTS devices are used most frequent in power systems [5].

An SVC is a device that is shunt-connected to a bus and injects or absorbs reactive power $Q_{\mathrm{SVC}}$ to control the voltage $V_{\mathrm{SVC}}$ at the bus to which it is connected [6]. The SVC is modeled as a shunt-connected variable susceptance (as in Fig. 2(a)) for which the injected reactive power $Q_{\mathrm{SVC}}$ is

$$
Q_{\mathrm{SVC}}=-V_{\mathrm{SVC}}^{2} B_{\mathrm{SVC}},
$$

where $B_{\mathrm{SVC}}$ is the variable effective susceptance of the device. The control input $B_{\mathrm{SVC}}$ is limited to the domain

$$
B_{\mathrm{SVC}, \min } \leq B_{\mathrm{SVC}} \leq B_{\mathrm{SVC}, \max },
$$

where the values of $B_{\mathrm{SVC} \text {, min }}$ and $B_{\mathrm{SVC} \text {, max }}$ are determined by the size of the device.

A TCSC is a device connected in series with a transmission line. It can change the line reactance $X_{\text {line }}$ and therefore is able to control the active power flowing over the line [6]. The device is modeled as a variable reactance $X_{\text {TCSC }}$ connected in series with the line, as in Fig. 2(b). The total reactance $X_{\text {line }}$ of the line including the TCSC is therefore

$$
X_{\text {line }}=X+X_{\text {TCSC }} \text {, }
$$

where $X$ is the reactance of the line without the TCSC installed. The reactance $X_{\mathrm{TCSC}}$ is limited to the domain

$$
X_{\mathrm{TCSC}, \min } \leq X_{\mathrm{TCSC}} \leq X_{\mathrm{TCSC}, \max }
$$

where the values of $X_{\mathrm{TCSC} \text {, min }}$ and $X_{\mathrm{TCSC} \text {,max }}$ are determined by the size of the TCSC device and the characteristics of the line in which it is placed, since due to the physics the allowed compensation rate of the line $X_{\mathrm{TCSC}} / X$ is limited [7].

\section{OPTIMAL POWER FLOW CONTROL PROBLEM}

Our OPF objective is to improve the system security through

1) minimization of deviations of bus voltages from given references to improve the voltage profile,

2) minimization of active power losses,

3) and preventing lines from overloading, by choosing appropriate settings for the FACTS devices. These goals are captured by the objective function

$$
\begin{array}{r}
f(\cdot)=\sum_{i=1}^{n}\left(V_{i}-V_{\mathrm{ref}, i}\right)^{2}+\sum_{(i, j) \in \mathcal{I}} P_{\text {loss }, i j}\left(V_{i}, \theta_{i}, V_{j}, \theta_{j}\right) \\
+\sum_{(i, j) \in \mathcal{I}}\left(\frac{S_{i j}\left(V_{i}, \theta_{i}, V_{j}, \theta_{j}\right)}{S_{i j, \max }}\right)^{2},
\end{array}
$$

where $n$ is the number of buses in the network, where for each bus $i \in\{1, \ldots, n\}, V_{i}$ and $\theta_{i}$ denote voltage magnitude and angle, where $\mathcal{I}$ denotes all $(i, j), i \in\{1, \ldots, n\}, j \in\{, \ldots, n\}$ for which there is a line between bus $i$ and $j$, and where for each $(i, j) \in \mathcal{I}, S_{i j}(\cdot)$ is the apparent power flow with maximum $S_{i j, \max }$ and $P_{\text {loss }, i j}(\cdot)$ the active power loss.

To determine the values of the variables involved in the objective function, a model of the network is used. The model of the network consists of the power flow equations coming forth from the models of the generators, loads, power lines, and FACTS devices as described in Sect. II, augmented with relations to compute $S_{i j}(\cdot)$ and $P_{\text {loss }, i j}(\cdot)$.

Denoting by $x$ all variables involved, the model of the network and additional bound constraints are combined into equality constraints $g(x)=0$ and inequality constraints $h(x) \leq 0$, such that the overall OPF problem involves minimizing $f(x)$ over $x$, subject to $g(x)=0$ and $h(x) \leq 0$. The solution of this optimization problem yields the settings for the FACTS devices that minimize the objective function, while taking into account the constraints. In theory such a control problem may be solvable; in practice this may be intractable or impossible, due to the large scale of the problem or inaccessibility of all actuators and sensors by a single controller. Instead, multiple smaller areas have to be considered and multi-area control has to be employed.

\section{NON-OVERlapPing MUlTi-AREA OPF CONTROL}

In multi-area OPF control, the overall OPF problem is decomposed into several subproblems each associated with an area. These subproblems are then solved in an iterative procedure. For this purpose, various decomposition techniques have been proposed over the years, mainly having their foundation in Lagrange and augmented Lagrange theory. A comparison on the performance in power systems of a selection of these methods is given in [2], [8], [9], [10].

One of the existing approaches is the modified Lagrange decomposition approach, proposed in [11] and applied to OPF in [3]. This approach makes for each area $p$ a distinction between local and external variables, where the local variables are the decision variables of area $p$ and the external variables are the decision variables of other areas. As we will see in the following, constraints that include local as well as external variables are interconnecting constraints and are moved as soft constraints into the objective function of one area, while keeping them explicit as hard constraints in the constraint set of another area. In each iteration, an area obtains values for the Lagrange multipliers of the interconnecting constraints that it considers as hard constraints. These values are communicated 
to the neighboring areas and used in their objective functions to penalize the corresponding soft constraints. The overall objective function is completely assigned to each area, although with the external variables fixed. We elaborate on this in the following.

The decomposition concept for constraints and objective function is based on the first-order optimality conditions for the overall problem and the subproblems. Applying this decomposition, the first-order optimality conditions for all areas combined are equivalent to the first-order optimality conditions for the overall problem [11]. In the following we focus on equality constraints, noting that inequality constraint can be transformed into equality constraints using slack variables.

The classification of equality constraints as hard or soft for a particular area in a multi-area power network is done in the following way: equality constraints are included as hard constraints in the area where the bus to which this equation is assigned, is located, and taken into account in the objective function of the other areas as soft constraints.

The procedure to set up the subproblems then is as follows:

1) Determine which buses, and thus which variables, are included in which area, defined, e.g., by given control regions or by sensitivity analysis.

2) Assign the overall objective function to each area $p$ and define the external variables as fixed.

3) For each bus $i$ in each area $p$, set up the power flow equality constraints and include them into the constraint set of area $p$.

4) Determine for each constraint whether it is an interconnecting constraint $g_{p \text {,int }}\left(x_{p}, x_{p_{\mathrm{e}}}\right)$ which involves local variables $x_{p}$ as well as external variables $x_{p_{\mathrm{e}}}$ or whether it is a constraint $g_{p}\left(x_{p}\right)$ only using local variables.

5) Include $g_{p \text {,int }}\left(x_{p}, x_{p_{\mathrm{e}}}\right)$ in each area $m$ as a soft constraint in the objective function for which a variable $x_{m}$ appears as external variable in this equation.

For $M$ areas, the subproblem solved by area $p \in$ $\{1, \ldots, M\}$ with decision variables $x_{p}$ at a particular iteration step is therefore given by

$$
\min _{x_{p}} f\left(x_{p}, \bar{x}_{p_{\mathrm{e}}}\right)+\left(\bar{\lambda}_{p_{\mathrm{e}}, \text { int }}\right)^{\mathrm{T}} g_{p_{\mathrm{e}}, \text { int }}\left(x_{p}, \bar{x}_{p_{\mathrm{e}}}\right)
$$

subject to

$$
\begin{aligned}
g_{p, \text { int }}\left(x_{p}, \bar{x}_{p_{\mathrm{e}}}\right) & =0 \\
g_{p}\left(x_{p}\right) & =0
\end{aligned}
$$

where the subscript $p$ denotes local variables and equations associated with buses in area $p$. The subscript $p_{\mathrm{e}}$ is used accordingly for external variables and equations associated with external buses. The bar and subscript $p_{\mathrm{e}}$ notation for a variable, e.g., $\bar{v}_{p_{\mathrm{e}}}$, indicates that the value of $v$ is set to the value determined for $v$ in the previous iteration. The subscript int indicates interconnecting constraints that include local variables as well as external variables. The variables $\lambda_{p_{\mathrm{e}} \text {,int }}$ are the Lagrange multipliers for the interconnecting constraints $g_{p_{\mathrm{e}} \text {,int }}$ included as soft constraints in area $p$ and as hard constraints in the area where the bus to which this constraint is associated to is located.

Using this problem setup for each area, the outline of the scheme to determine the settings of the manipulated variables, e.g., FACTS devices, is as follows:

1) Each area $p$ initializes its variables $x_{p}$ and $\lambda_{p \text {,int }}$ by setting the variables for voltage magnitudes and angles and the manipulated variable to the current steady-state values and the Lagrangian multipliers to zero.

2) Iteration counter $s$ is set to 1 .

3) Given $\bar{x}_{p_{\mathrm{e}}}$ and $\bar{\lambda}_{p_{\mathrm{e}} \text {,int }}$ from the initialization or iteration step $s-1$, each area $p \in\{1, \ldots, M\}$ solves in parallel with the other areas its subproblem given by equations (6)-(8) to obtain $x_{p}$ and $\lambda_{p \text {,int }}$ for iteration $s$.

4) The areas exchange the requested values resulting from their optimization problem with their neighbors.

5) Unless a stopping condition is satisfied, e.g., the absolute changes in all variables from step $s-1$ to $s$ are smaller than a pre-defined threshold, the next iteration is started by increasing $s$ and going back to step 3 .

In order to speed up the computations, in the original method only one Newton-Raphson step is performed in solving the subproblem in step 3, instead of determining the actual optimal solution of the subproblem [11]. In [11] a proof is given that shows that when applying an interior point algorithm in combination with a conjugate gradient method, the multi-area control converges to the overall optimal solution. In our approach, we solve the optimization problem completely as the computation time for one iteration is not considered here and convergence is expected to be faster.

The advantage of the method in [11] over the augmented Lagrange methods as discussed is that no parameter tuning is necessary. Only the state variables and the Lagrange multipliers have to be initialized. A shortcoming is that this method, as well as other methods, requires that the network is decomposable into non-overlapping areas as it is assumed that each variable can be assigned to either one of the areas. However, in case of overlapping areas, certain variables and constraints are included in more than one area and the identification of local and external constraints is not straightforward any more. Therefore, the method is not directly applicable for our purpose. In the following, we consider an extension of this method to overlapping areas.

\section{EXTENSION TO OVERLAPPING AREAS}

Now we extend the approach for non-overlapping areas to overlapping areas. For multi-area control in power systems, areas are overlapping areas when at least one bus, and thus some variables, cannot be assigned uniquely to one particular area, but are common to at least two areas, contrary to nonoverlapping areas, for which no bus is included at the same time in multiple areas, see Fig. 1. Overlapping areas thus share a common area, consisting of those buses that are part of multiple areas. In the case of overlapping areas, several difficulties concerning the choice of the decision variables, the constraints, and the objective functions have to be overcome. 


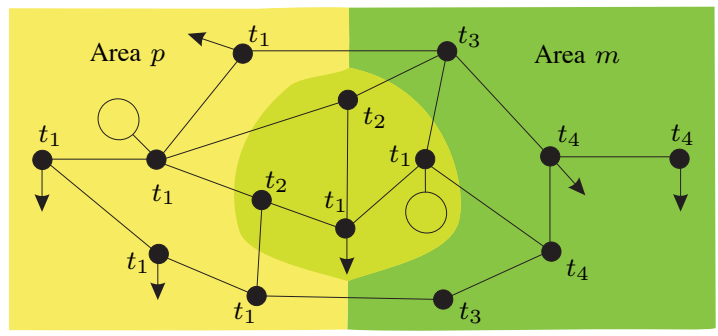

Fig. 3. Illustration of different bus types.

\section{A. Decision variables}

From the point of view of a particular area $p$ three types of buses can be determined:

1) local buses: buses which are only included in area $p$;

2) common buses: those buses included in area $p$ and some other area $m$, hence, located in the common area;

3) external buses: those busses not included in area $p$.

The terms local, common, and external are also used for the variables associated with the respective buses. The local variables for area $p$ are denoted by $x_{p}$, the common variables by $x_{p_{\mathrm{c}}}$, and the external variables by $x_{p_{\mathrm{e}}}$.

Each area has its local variables as decision variables in the OPF problem. Furthermore, also the common variables are considered as its decision variables. In the course of the iteration process, the values of these common variables are exchanged among the areas. The external variables of an area are not considered decision variables, but instead assumed given by another area.

The difficulty for multi-area OPF for overlapping areas arises from the common variables. Even though we assume that the areas have the same objective with respect to these variables, combined with the objective for their local variables, this might result in conflicting intentions for the common variables.

\section{B. Constraints}

There are constraints that depend on common variables and possibly also on local or external variables. In power systems, such constraints correspond to the power flow equations for the buses in the common area and buses in any of the areas which are connected to at least one bus in the common or in an other area.

In order to classify the types of constraints, we first classify the types of buses. For multi-area OPF with overlapping areas, four different types of buses are distinguished. In Fig. 3, an illustration of these types from the view point of area $p$ is given. The bus types $t_{1}-t_{4}$ can be described as:

1) bus type $t_{1}$ : all local buses and in addition, common buses that are not connected to any local bus;

2) bus type $t_{2}$ : common buses that are connected to at least one local bus;

3 ) bus type $t_{3}$ : external buses that are connected to at least one local bus;
TABLE I

TREATMENT OF CONSTRAINTS ASSOCIATED WITH DIFFERENT BUS TYPES FOR AREA $p$

\begin{tabular}{|c|l|l|}
\hline type & bus location & how to deal with constraints \\
\hline \hline$t_{1}$ & local, common & $g_{t_{1}}\left(x_{p}, \bar{x}_{p_{\mathrm{e}}}, x_{p_{\mathrm{c}}}\right)$ \\
\hline$t_{2}$ & common & $g_{t_{2}}\left(x_{p}, \bar{x}_{p_{\mathrm{e}}}, x_{p_{\mathrm{c}}}\right)$ \\
& & $f_{p}=\cdots+\bar{\lambda}_{p_{\mathrm{c}}} g_{t_{2}}\left(x_{p}, \bar{x}_{p_{\mathrm{e}}}, \bar{x}_{p_{\mathrm{c}}}\right)$ \\
\hline$t_{3}$ & external & $f_{p}=\cdots+\bar{\lambda}_{p_{\mathrm{e}}} g_{t_{3}}\left(x_{p}, \bar{x}_{p_{\mathrm{e}}}, \bar{x}_{p_{\mathrm{c}}}\right)$ \\
\hline$t_{4}$ & external & - \\
\hline
\end{tabular}

4) bus type $t_{4}$ : external buses that are connected to only external and common buses.

Each of the bus types yields a different way of dealing with the constraints associated with that bus in the OPF formulation for the area. In general, if a constraint is included in one area using fixed values for the external variables, it is included in the objective function of another area with contrary variables fixed and weighted with the Lagrange multiplier for this constraint given from the first area. So, for a particular area, its constraints of type $t_{1}$ and $t_{2}$ are included as hard constraints with external variables fixed, while its constraints of $t_{2}$ and $t_{3}$ are included as soft constraints in the objective function with external and common variables fixed. Constraints associated with buses of type $t_{4}$ are not taken into account in the considered area. Table I gives the overview of how constraints associated to different bus types are taken into account.

\section{Objective function}

From the point of view of area $p$, the overall objective function consists of terms that involve only local variables, common variables, and external variables, and possibly combinations. E.g., if the deviations of voltages from a given reference value are minimized, the objective function includes, among others, terms that are dependent on only common variables. When active power losses are minimized, both common and external variables can be involved in objective function terms.

In the case of overlapping areas, the overall objective function is decomposed such that it holds that the gradient of the overall objective function is equal to the sum of the gradients of the objective functions of the areas, i.e.,

$$
\frac{\partial f}{\partial x}=\sum_{p=1}^{M} \frac{\partial f_{p}}{\partial x} .
$$

The concept used is to equally divide the terms including only common variables and assign them to the involved areas. Terms that use local variables from one area and variables from the common area are only included in the area that includes all variables, and terms that use variables from both areas are fully included in both areas.

Table II shows how terms depending on local, common, external variables, and combinations of these are taken into account in the decomposed system fulfilling criteria (9). The number of areas that include the common variables appearing in the considered term is denoted by $a$. 
TABLE II

DECOMPOSITION OF THE OBJECTIVE FUNCTION FOR AREA $p$

\begin{tabular}{|l|l|}
\hline term in overall $f(\cdot)$ & how to include in $f_{p}(\cdot)$ \\
\hline \hline$f_{1}\left(x_{p}\right)$ & $f_{p}(\cdot)=\cdots+f_{1}\left(x_{p}\right)$ \\
\hline$f_{2}\left(x_{p}, x_{p_{\mathrm{c}}}\right)$ & $f_{p}(\cdot)=\cdots+f_{2}\left(x_{p}, x_{p_{\mathrm{c}}}\right)$ \\
\hline$f_{3}\left(x_{p_{\mathrm{e}}}, x_{p_{\mathrm{c}}}\right)$ & $f_{p}(\cdot)=\cdots+0$ \\
\hline$f_{4}\left(x_{p_{\mathrm{c}}}\right)$ & $f_{p}(\cdot)=\cdots+\frac{1}{a} f_{4}\left(x_{p_{\mathrm{c}}}\right)$ \\
\hline$f_{5}\left(x_{p}, x_{p_{\mathrm{e}}}\right)$ & $f_{p}(\cdot)=\cdots+f_{5}\left(x_{p}, \bar{x}_{p_{\mathrm{e}}}\right)$ \\
\hline
\end{tabular}

If the objective function is decomposed like this and the constraints are taken into account as given in the previous section, the first-order optimality conditions for the overall problem and the subproblems show that this decomposition yields the same optimal solution for the overall problem as for the decomposed problem.

\section{Extended scheme}

Having defined how constraints and objective function are formulated in the overlapping case, the adapted scheme is as follows:

1) Determine which buses, and thus which variables, are included in which area and distinguish between local, common, and external variables.

2) Define the objective function of each area $p$ including the terms of the overall objective function as defined in Table II.

3) For each bus $i$ and each area $p$, set up the power flow equality constraints and include them into the constraint set of area $p$.

4) Determine the type $t_{1}, \ldots, t_{4}$ for each bus.

5) For buses of type $t_{2}$ and $t_{3}$ include the constraints associated with these buses according to Table I as soft constraints into the objective function.

Hence, the structure of the subproblem for each area $p$ has been set up and can be used to formulate the subproblems for use in a similar scheme as given for the non-overlapping case.

\section{Simulation RESUlts}

Simulations are carried out on the IEEE 57-bus grid with additional FACTS devices installed at various locations [12]. In order to find an interesting and meaningful situation for FACTS control, the grid was adapted by placing an additional generator at bus 30 leading to increased power flows in the center of the grid.

Various test scenarios with different FACTS devices and area definition have been examined. Here we present two representative scenarios. The areas used in these scenarios are shown in Fig. 4. It can be seen that these areas are overlapping, since there are several buses that are included in both areas.

The objective function for the overall system is defined as in (5) and decomposed as elaborated in Sect. V-C. The controllers of each area use the SNOPT solver of Tomlab to solve their subproblems at each iteration step.

\section{A. Scenario 1: Control of SVCs}

In the first scenario, two SVCs are present. The SVCs are placed at (local) buses 14 and 29. As the SVCs are mainly

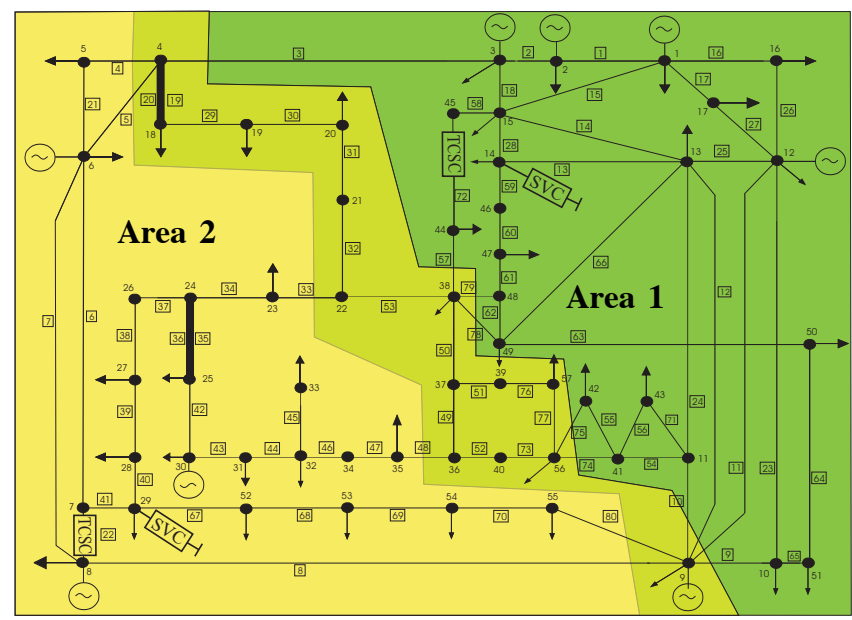

Fig. 4. IEEE 57-bus system with decomposition into 2 areas. Scenario 1: SVCs at buses 14 and 29, scenario 2: TCSCs in lines 22 and 72.

used to influence the voltage profile, the line limits are chosen such that no line is at the risk of being overloaded.

Fig. 5 shows the convergence of the SVC device settings over the iterations. As can be seen, the settings of the SVC devices converge within only a few iterations to the final values, which are equal to the values obtained from an overall optimization. Fig. 6 shows the evolution of the deviations between the values determined by both areas for the voltage magnitudes and angles at some common buses again indicating fast convergence.

\section{B. Scenario 2: Control of TCSCs}

In the second scenario, two TCSC devices are installed in lines 72 and 22. Since TCSCs are mainly used to influence active power flows and to resolve congestion, the line limits are chosen such that lines 7 and 60 are overloaded in the base case when the FACTS devices are set out of operation.

The results for the TCSC device settings and the difference between the voltage magnitudes and angles for some common buses over the iterations are given in Figs. 7 and 8, respectively. The line reactance of line 72 is $0.1242 \Omega$ yielding an upper compensation limit of $0.02484 \Omega$ (see Sect. II). Thus, the controller of area 1 sets the TCSC to its upper limit for the first few iterations. But after some additional iterations, the TCSC settings converge to their final value which are again equal to the values obtained from the overall optimization.

In Fig. 9, the line loadings of lines 7 and 60, the lines which are overloaded without FACTS devices, are shown. Line 7 is immediately brought below its limit whereas for line 60 , the loading approaches $100 \%$ in the course of the optimization process.

\section{CONCLUSIONS AND FUTURE WORK}

A method for decomposition of control problems assuming non-overlapping areas is extended to applications with overlapping areas. Various aspects concerning state variables, constraints and objective function are considered and taken into account in the extended method. Simulations show fast 


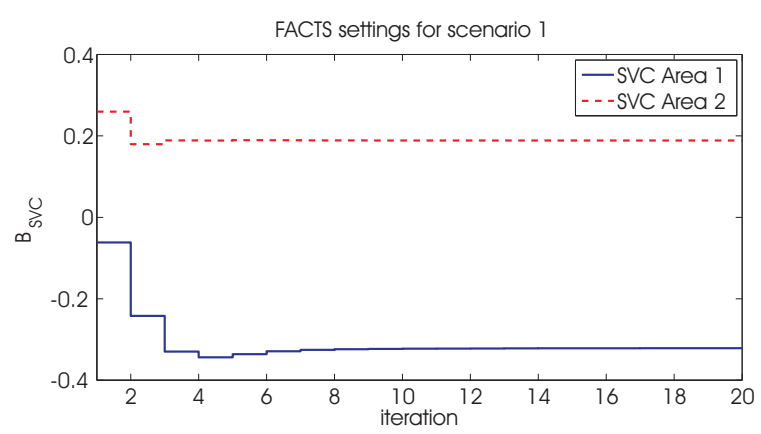

Fig. 5. FACTS device settings for SVCs at buses 14 and 29.
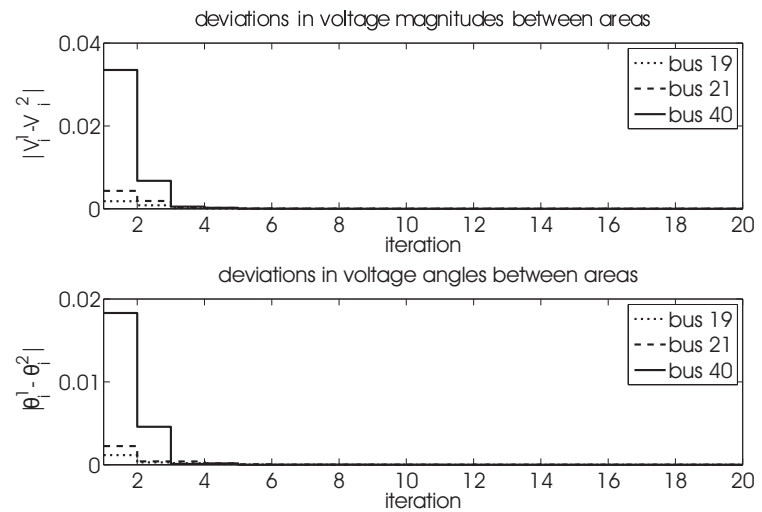

Fig. 6. Voltage magnitudes and angles for buses 19, 21, 40 for scenario 1 with SVCs at buses 14 and 29.

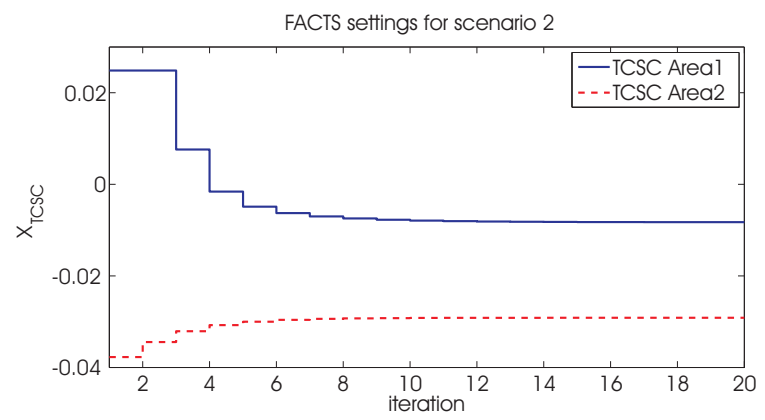

Fig. 7. FACTS device settings for TCSCs in lines 22 and 72
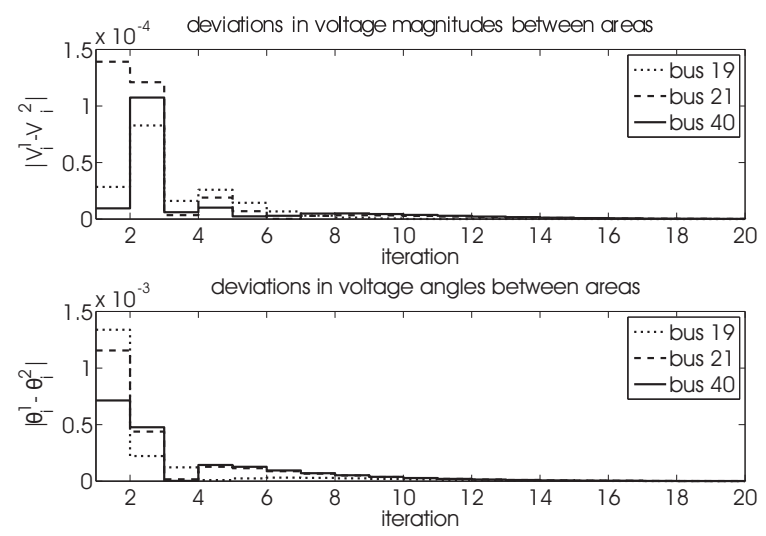

Fig. 8. Voltage magnitudes and angles for buses 19, 21, 40 for scenario 2 with TCSCs in lines 22 and 72.

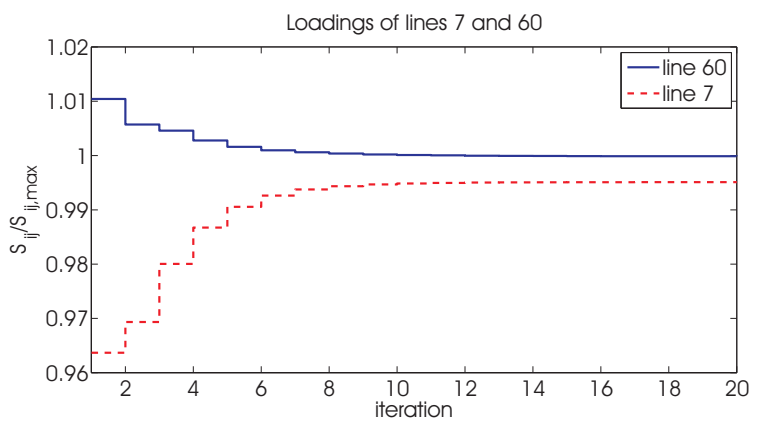

Fig. 9. Line loadings of lines 7 and 60 for scenario 2 with TCSCs in lines 22 and 72.

convergence to overall optimal values for problems involving SVC and TCSC devices.

Future work will explore the use of reduced area models determined by sensitivity analysis in which the models of the areas do not fully cover the whole network.

\section{ACKNOWLEDGMENTS}

This research was supported by the project "HYbrid CONtrol: Taming Heterogeneity and Complexity of Networked Embedded Systems (HYCON)", contract number FP6-IST-511368, the project "Coordinated control of FACTS devices", supported by ABB Switzerland, the project "Multi-agent control of large-scale hybrid systems" (DWV.6188) of the Dutch Technology Foundation STW, and the BSIK project "Next Generation Infrastructures (NGI)".

\section{REFERENCES}

[1] P. Kundur. Power System Stability and Control. McGraw Hill, New York, 1994.

[2] B. H. Kim and R. Baldick. A comparison of distributed optimal power flow algorithms. IEEE Transactions on Power Systems, 15(2):599-604, 2000.

[3] F. J. Nogales, F. J. Prieto, and A. J. Conejo. Multi-area AC optimal power flow: A new decomposition approach. In Proc. of the 13th Power Systems Control Conference (PSCC), pages 1201-1206, Trondheim, Germany, 1999.

[4] G. Glanzmann and G. Andersson. FACTS control for large power systems incorporating security aspects. In Proc. of X SEPOPE, Florianopolis, Brazil, 2006.

[5] A. Edris, R. Adapa, M. H. Baker, L. Bohmann, K. Clark, K. Habashi, L. Gyugyi, J. Lemay, A. S. Mehraban, A. K. Meyers, J. Reeve, F. Sener, D. R. Torgerson, and R. R. Wood. Proposed terms and definitions for flexible AC transmission system (FACTS). IEEE Transactions on Power Delivery, 12(4):1848, 1997.

[6] N. G. Hingorani and L. Gyugyi. Understanding FACTS concepts and technology of flexible AC transmission systems. IEEE Press, New York, 2000.

[7] G. Glanzmann and G. Andersson. Using FACTS devices to resolve congestions in transmission grids. In Proc. of CIGRE/IEEE PES International Symposium, San Antonio, USA, 2005.

[8] M. Arnold and S. Knöpfli. Multi-Area Control in Electric Power Systems. Master thesis, ETH Zürich, 2006.

[9] R. R. Negenborn, B. De Schutter, and J. Hellendoorn. Multi-agent model predictive control for transportation networks: Serial versus parallel schemes. In Proceedings of the 12th IFAC Symposium on Information Control Problems in Manufacturing (INCOM 2006), pages 339-344, Saint-Etienne, France, 2006.

[10] M. Arnold, S. Knöpfli, and G. Andersson. Improvement of OPF decomposition methods applied to multi-area power systems. In Proc. of PowerTech 2007, Lausanne, Switzerland, 2007.

[11] A. J. Conejo, F. J. Nogales, and F. J. Prieto. A decomposition procedure based on approximate newton directions. Mathematical Programming, Series A, 93(3):495-515, December 2002.

[12] Power Systems Test Case Archive. Parameters of the IEEE 57-bus grid. http://www.ee.washington.edu/research/pstca/. 